\title{
Síntesis de nanopartículas de hidroxiapatita y su efecto en plántulas de Raphanus sativus
}

\section{Synthesis of hydroxyapatite nanoparticles and its effect on Raphanus sativus seedlings}

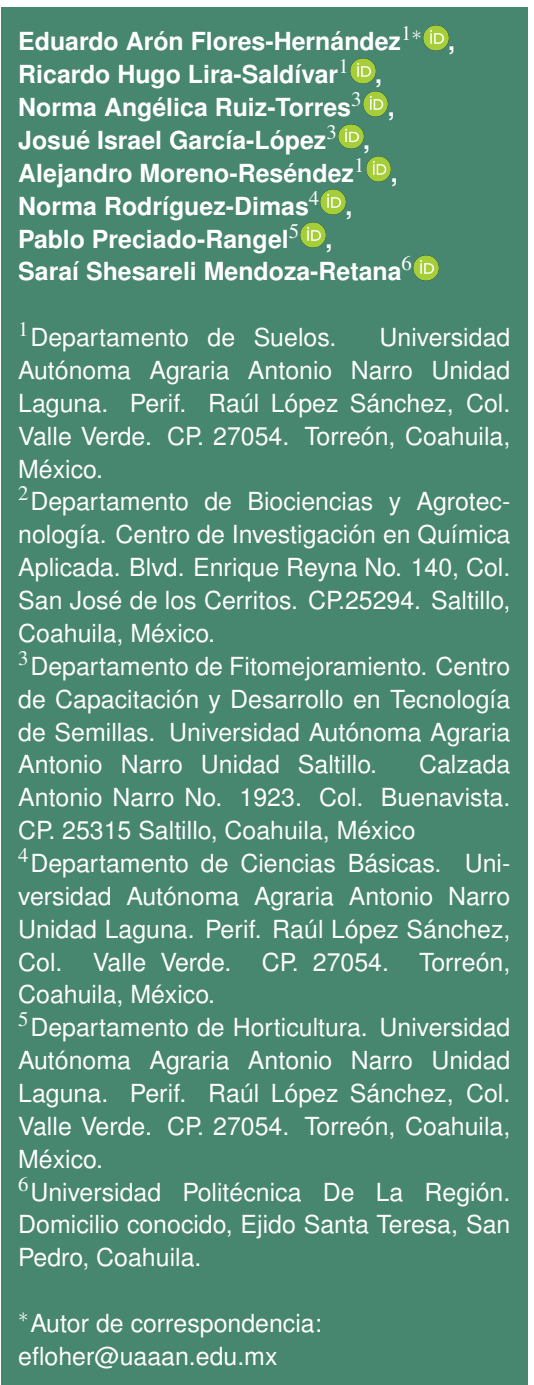

Artículo científico Recibido: 20 de octubre 2020 Aceptado: 24 de febrero 2021

Como citar: Flores-Hernández EA, LiraSaldivar RH, Ruiz-Torres NA, García-López Jl, Moreno-Reséndez A, Rodríguez-Dimas N, Preciado-Rangel P, Mendoza-Retana SS (2021) Síntesis de nanopartículas de hidroxiapatita y su efecto en plántulas de Raphanus sativus. Ecosistemas y Recursos Agropecuarios 8(1): e2747. 10.19136/era.a8n1.2747
RESUMEN. Recientemente se ha reportado el potencial de los nanofertilizantes para incrementar rendimientos de los cultivos. Las nanopartículas de hidroxiapatita (nHAp) se aplican como fertilizantes a las plantas por su alto contenido en $\mathrm{P}$ y Ca $\left(\mathrm{Ca}_{10}\left(\mathrm{PO}_{4}\right)_{6}(\mathrm{OH})_{2}\right)$, pero hay escasa información al respecto. El objetivo del trabajo fue producir nHAp sintético, caracterizarlo y evaluar su efecto como nutriente en el desarrollo de plántulas de rábano (Raphanus sativus) variedad Champion. La síntesis de nHAp se realizó mediante una solución acuosa de hidróxido de calcio $\left[\mathrm{Ca}(\mathrm{OH})_{2}\right]$ y ácido ortofosfórico $\left(\mathrm{H}_{3} \mathrm{PO}_{4}\right)$. Para analizar su efecto biológico, se estableció un bioensayo con siete tratamientos en semillas de rábano con las siguientes concentraciones: control (agua destilada, T1); 25 (T2); 50 (T3); 100 (T4); 200 (T5); 500 (T6) y $1000 \mathrm{mg} \mathrm{L}^{-1}$ (T7); las plántulas se desarrollaron hasta los siete días después de la siembra. Los resultados muestran que una concentración de $1000 \mathrm{mg} \mathrm{L}^{-1}$ redujo la germinación en $14,4 \%$ en comparación con el tratamiento control $\left(0 \mathrm{~mL}^{-1}\right)$. Mientras que el vigor de las plántulas se redujo $11.2 \%$ con $500 \mathrm{mg} \mathrm{L}^{-1}$. La plúmula y radícula no mostraron diferencias significativas por los tratamientos con respecto al control, sin embargo, se observó una diferencia estadística con las dosis de 25 y $200 \mathrm{mg} \mathrm{L}^{-1}$, al inhibir y promover respectivamente el crecimiento de las plántulas. Se observó que altas concentraciones de nHAp generaban efectos adversos en las semillas. Finalmente, las nHAp estimularon la producción de biomasa.

Palabras clave: Nanotecnología, nanofertilizante, radícula, semillas, vigor.

ABSTRACT. The potential of nanofertilizers to increase crop yields has recently been reported. Hydroxyapatite nanoparticles (nHAp) are applied as fertilizers to plants due to their high content of $\mathrm{P}$ and $\mathrm{Ca}\left(\mathrm{Ca}_{10}\left(\mathrm{PO}_{4}\right)_{6}(\mathrm{OH})_{2}\right)$, but there is little information in this regard. The objective of the work was to produce synthetic $\mathrm{nHAp}$, characterize it and evaluate its effect as a nutrient in the development of seedlings of radish (Raphanus sativus) variety Champion. The synthesis of nHAp was carried out using an aqueous solution of calcium hydroxide $\left[\mathrm{Ca}(\mathrm{OH})_{2}\right]$ and orthophosphoric acid $\left(\mathrm{H}_{3} \mathrm{PO}_{4}\right)$. To analyze its biological effect, a bioassay was established with seven treatments on radish seeds with the following concentrations: control (distilled water, T1); 25 (T2); 50 (T3); 100 (T4); 200 (T5); 500 (T6) and $1000 \mathrm{mg} \mathrm{L}^{-1}$ (T7); the seedlings developed up to seven days after sowing. The results show that a concentration of $1000 \mathrm{mg} \mathrm{L}^{-1}$ reduced germination by $14.4 \%$ compared to the control treatment $\left(0 \mathrm{~mL}^{-1}\right)$. While the vigor of the seedlings was reduced $11.2 \%$ with $500 \mathrm{mg} \mathrm{L}^{-1}$. The plumule and radicle did not show significant differences by the treatments with respect to the control, however, a statistical difference was observed with the doses of 25 and $200 \mathrm{mg}$ $\mathrm{L}^{-1}$, when inhibiting and promoting the growth of the seedlings, respectively. High concentrations of $\mathrm{nHAp}$ were found to have adverse effects on seeds. Finally, the nHAp stimulated biomass production.

Key words: Nanotechnology, nanofertilizer, radicle, seeds, vigor. 


\section{INTRODUCCIÓN}

Para atender la demanda de alimentos a nivel mundial, en la década de 1960 se inició la Revolución Verde. Durante este periodo se promovieron los fertilizantes sintéticos, usados cada vez en mayor cantidad para incrementar los rendimientos agrícolas (Pazos-Rojas et al. 2016). Pero las reservas de roca fosfórica que se utilizan para elaborar los fertilizantes fosfatados son finitas, estimándose que las reservas se agotaran en los próximos 50 a 125 años con el ritmo de explotación actual (Kabiri et al. 2020; Cordell y White 2015). Como consecuencia a medida que continua la extracción de roca fosfórica, la calidad del material de los yacimientos disminuye, los costos de producción aumentan y la problemática del futuro suministro de $\mathrm{P}$ parece recibir poca atención (Cordell et al. 2009). Aunado a lo anterior, el empleo de agroquímicos sintéticos convencionales incrementa la presencia de metales pesados y agentes tóxicos, lo que deteriora la calidad del suelo, además de disminuir su fertilidad natural (Méndez-Argüello y Lira-Saldívar 2019). Como una alternativa para mitigar la futura escasez de P, se ha propuesto el uso de la roca fosfórica sintetizada en laboratorio (hidroxiapatita), la cual puede dosificarse de forma directa al cultivo como una suspensión de nanopartículas para mejorar su eficiencia de aplicación y absorción, lo que aumenta la disponibilidad del $\mathrm{P}$ para las plantas, al evitar su fijación en el suelo (Banotra et al. 2017). Las propiedades físicas y químicas de los materiales a escala nanométrica son diferentes a las del material a granel (Saleh 2020). El uso de nanofertilizantes para la liberación y control preciso de nutrientes permite el desarrollo de una agricultura sostenible y compatible con el ambiente, ya que se reduce la cantidad de insumos y se minimizan las pérdidas de nutrientes (Predoi et al. 2020).

La principal ventaja de usar nHAp como nanofertilizante, con respecto a otros nanomateriales, es que son reconocidas por su biocompatibilidad y biodegradabilidad intrínseca (Ramesh et al. 2018). Por lo tanto; cuando se considera su aplicación en campo abierto, el perfil biológico de la nHAp no debe plantear ninguna preocupación sobre la salud humana y ambiental (Marchiol et al. 2019). Con respecto a la aplicación de la nHAp en la agricultura, Rane et al. (2015) documentaron que este tipo de nanopartículas (NPs) pueden mejorar la fisiología de plantas de maíz al aumentar el contenido de clorofila, el crecimiento vegetativo, la proliferación de raíces y el rendimiento. Al respecto, Montalvo et al. (2015) utilizaron las HAp NPs en plantas de trigo, reportaron que ese tipo de NPs pueden actuar como fertilizante fosfatado, ya que su tamaño permite que se muevan con facilidad en el suelo, penetren por las raíces y fluyan por el xilema, mediante el flujo masivo de agua creado por la transpiración.

En un estudio realizado con plantas de lechuga (Lactuca sativa) se reporta que la biomasa seca de plantas cultivadas en suelos calcáreos incrementa con la aplicación del P contenido en los nHAp, este fue el método más efectivo que la fuente ordinaria de fertilizante de $\mathrm{H}_{3} \mathrm{PO}_{4}-\mathrm{P}$ (Taşkın et al. 2018). En plantas de tomate (Solanum lycopersicum), Marchiol et al. (2019) determinaron que la elongación radicular debido a la aplicación de nHAp fue estimulada de forma significativa y que las plantas de tomate cultivadas en hidroponía no sufrieron efectos fitotóxicos. Por lo que bajas concentraciones de HAp NPs podrían usarse como aportadoras de $\mathrm{P}$ y $\mathrm{Ca}$, así como de otros elementos y moléculas nutritivas para las plantas. Con respecto a la potencial fitotoxicidad de las HAp NPs, hay reportes que afirman que las dosis elevadas de NPs metálicas inducen dicho efecto y conllevan un posible riesgo para el crecimiento de las plantas, en las cuales se han observado efectos citotóxicos y genotóxicos debido al estrés oxidativo que generan las especies reactivas de oxígeno (ERO) (Antisari et al. 2018). Mientras que Rui et al. (2017) al evaluar nanopartículas de plata (NPsAg) en cacahuate (Arachis hypogaea L.), reportaron que la dosis de $2000 \mathrm{mg} \mathrm{Kg}^{-1}$ inhibió el crecimiento de las plantas y la formación de vainas. Otros estudios con NPs de óxidos metálicos como $\mathrm{CuO}, \mathrm{NiO}, \mathrm{TiO}_{2}$, $\mathrm{Fe}_{2} \mathrm{O}_{3}$ y $\mathrm{Co}_{3} \mathrm{O}_{4}$ a diferentes dosis, reportan una reducción en la germinación de semillas de lechuga, rábano y pepino; esto se atribuye a la adsorción de éstas sobre las superficies de las semillas y la liberación de iones metálicos, en dosis mayores los 
efectos tóxicos de las NPs varían con el tipo de cultivo y el tamaño de la semilla (Rizwan et al. 2017). Por su parte Jain et al. (2017), evaluaron la fitotoxicidad de NPs de $\mathrm{ZnO}$ en semillas de mijo (Pennisetum glaucum) y tomate (Solanum lycopersicum), encontraron que una concentración de $1000 \mathrm{mg} \mathrm{L}^{-1}$ redujo la germinación en 18.3 y $60 \%$ para el mijo y tomate, respectivamente. Además de que las estructuras de las semillas de mijo (cutícula gruesa y testa lisa), les permitieron ser menos sensibles a las suspensiones con NPs de $\mathrm{ZnO}$, en comparación con las semillas de tomate, las cuales tienen testa delgada y rugosa.

Para las HAp NPs a la fecha existen pocos estudios que evalúen su impacto en la germinación y crecimiento de plántulas. Al respecto, Marchiol et al. (2019), reportan que la germinación de semillas de tomate no fue afectada por el aumento en la concentración de HAp NPs en un rango de 2 a $2000 \mathrm{mg} \mathrm{L}^{-1}$, mientras que la elongación de la raíz fue estimulada. Mientras que Ortiz et al. (2017) reportan que las nHAp no tiene efectos tóxicos en la germinación de semillas de $L$. sativa, por lo que la aplicación de las nHAp podría considerarse como proveedor de los macronutrientes $\mathrm{P}$ y $\mathrm{Ca}$. Pero ningún estudio ha abordado el efecto de las nHAp sobre la germinación, vigor y crecimiento de plántulas de $R$. sativus. Debido a esto, la hipótesis de este trabajo experimental fue, que las diferentes dosis de nHAp pueden provocar efectos variables en aspectos fisiológicos del rábano. Por lo anterior, el objetivo del presente trabajo fue producir HAp NPs sintéticas, caracterizarlas y evaluar su efecto como nutriente en el desarrollo de plántulas de rábano.

\section{MATERIALES Y MÉTODOS}

\section{Material vegetal}

La variedad de $R$. Sativus utilizada fue Champion (SAKATA ${ }^{\circledR}$, México, Zapopan Jalisco) de polinización libre, que genera bulbos uniformes de forma redonda y color rojo brillante, ideal para cultivarse el ciclo otoño-invierno.

\section{Síntesis de nanopartículas de hidroxiapatita}

La síntesis de las HAp NPs se realizó mediante el procedimiento descrito por Kottegoda et al. (2011), a partir de una solución acuosa de hidróxido de calcio $\left[\mathrm{Ca}(\mathrm{OH})_{2}\right]$ y ácido orto fosfórico $\left(\mathrm{H}_{3} \mathrm{PO}_{4}\right)$, con una relación estequiométrica de $\mathrm{Ca} / \mathrm{P}=1.67$. La mezcla se mantuvo en agitación constante y se calentó a 95 ${ }^{\circ} \mathrm{C}$ para activar la reacción química, como se muestra a continuación.

$$
6 \mathrm{H}_{3} \mathrm{PO}_{4}+10 \mathrm{Ca}(\mathrm{OH})_{2} \longrightarrow \mathrm{Ca}_{10}\left(\mathrm{PO}_{4}\right)_{6}(\mathrm{OH})_{2}+18 \mathrm{H}_{2} \mathrm{O}
$$

Una vez realizada la reacción de síntesis de nHAp, la solución resultante se dejó reposar durante $72 \mathrm{~h}$ hasta que el producto esperado se precipitó y formó la separación de fase, al final del tiempo indicado.

\section{Caracterización de las nHAp por difracción de Rayos X (XRD)}

Las $\mathrm{nHAp}$ se examinaron mediante un difractómetro XRD (Rigaku, Ultima IV $\AA)$ ). Los patrones de difracción de $X R D$ se registraron en un rango de $2 \theta$ de 10 a $80^{\circ}$, se obtuvo un tamaño de NPs en el rango de 10 a $45 \mathrm{~nm}$. El cálculo para obtener el tamaño promedio de las partículas de cristalita, se realizó mediante el software instalado en ese equipo con base al método Halder-Wagner (Izumi y Takuji 2015).

\section{Caracterización de las nHAp con microscopio electrónico de transmisión (TEM)}

La morfología y la microestructura de las $\mathrm{nHAp}$ se determinó con un microscopio FEI-TITAN 80300 kV (Fisher Scientific, Hillsboro ${ }^{\circledR}$ ), operado a un voltaje de aceleración de $300 \mathrm{kV}$. Las muestras para los análisis se prepararon al depositar y evaporar una gota de la solución coloidal en rejillas de encaje de carbono y cobre. El procesamiento de micrografías del TEM se interpretó mediante el software Digital Micrograph 3.7.0 (Gatan Software, Inc.). La descripción de las nHAp se realizó con imágenes de microscopía electrónica de transmisión y alta resolución (CTEM y HRTEM), así como por difracción de electrones (SAED). 
El análisis de la imagen del TEM para determinar el diámetro principal entre las NPs, se realizó al medir al menos 300 de ellas. La distribución del tamaño de partícula a partir de las imágenes CTEM, se realizó con el siguiente procedimiento: 1) con el software Image Pro-10 (Media Cibernetics Inc., Version 10.0.3) se midió el diámetro medio de más de 300 NPs; 2) con los datos obtenidos, se determinó la frecuencia absoluta para rangos de diámetros típicos; y 3) con los datos anteriores expresados en porcentaje se crearon los histogramas presentados.

\section{Tratamientos aplicados de HAp NPs}

Las suspensiones se prepararon con nanopartículas de un tamaño entre 10 y $45 \mathrm{~nm}$. La concentración inicial fue de $5000 \mathrm{mg} \mathrm{L}^{-1}$, en suspensión en agua desionizada estéril y dispersada durante 30 min con un sonicador Tianjing Autoscience (AS2060 Instrumental Factory ${ }^{\circledR}$ ). La suspensión se diluyó y se aplicaron siete tratamientos, los cuales fueron: control (agua destilada, T1); 25 (T2); 50 (T3); 100 (T4); 200 (T5); 500 (T6) y $1000 \mathrm{mg} \mathrm{L}^{-1}$ (T7).

\section{Germinación de semillas y crecimiento de plántu- las \\ En cada tratamiento se utilizaron 75 semillas} las cuales se colocaron en cajas Petri $(100 \times 15 \mathrm{~mm})$, sin división. Los tratamientos se aplicaron una sola vez durante la etapa de imbibición, para lo cual se añadieron $10 \mathrm{~mL}$ de suspensión de nHAp con una pipeta, en cada uno de los tratamientos. Para luego colocar las cajas Petri durante $4 \mathrm{~h}$ en una cámara de crecimiento para plantas [Equitec EGCS 3S, 301 $3 S H R$ (Equitek) $\left.{ }^{\circledR}\right]$, a $25 \pm 2{ }^{\circ} \mathrm{C}$ con un fotoperíodo de 16 horas luz y 8 horas de oscuridad. Completado el período de imbibición de las semillas, se sembraron tres réplicas de 25 semillas por tratamiento en papel Anchor (Seedburo Equipment Company), humedecido con agua destilada, las semillas se colocaron de forma horizontal, sobre el papel, con el embrión ubicado hacia abajo, enseguida se procedió a humedecer otro papel del mismo tamaño para cubrir las semillas. Posteriormente, se plegaron en un rollo de $4 \mathrm{~cm}$ de ancho, que se colocaron en cestas de polietileno de $20 \times 45 \mathrm{~cm}$ de ancho y alto, y se re- gresaron a la cámara de crecimiento durante siete días bajo las mismas condiciones de temperatura y fotoperíodo.

\section{Parámetros evaluados durante el experimento}

El bioensayo se desarrolló de acuerdo con las normas del ISTA (2004), las cuales consideran los siguientes parámetros: porcentaje de vigor y de germinación, longitud de plúmula y radícula $(\mathrm{cm})$, al finalizar el bioensayo se determinó la acumulación de biomasa seca en miligramos por plántula.

\section{Vigor de semillas (V\%)}

Al cuarto día después de la siembra, se realizó el primer conteo para la toma de datos de plántulas normales (plántulas que tienen bien desarrolladas la raíz y la plúmula, con desarrollo total de $2.0 \mathrm{~cm}$ en promedio). Para determinar el vigor de la semilla, se expresó el resultado en porcentaje.

Vigor de semilla $(V \%)=\frac{\text { plántulas normales }}{\text { número de semillas incubadas }} \times 100$

\section{Porcentaje de germinación (G\%)}

Se determinó a los siete días después de la siembra en el segundo conteo, para lo cual se consideró el conteo total de plántulas normales y el resultado se expresó en porcentaje.

$$
\begin{aligned}
& \text { Porcentaje de germinación }(G \%)= \\
& \frac{\text { número de semillas germinadas }}{\text { número de semillas incubadas }} \times 100
\end{aligned}
$$

\section{Longitud de plúmula y radícula}

La longitud de la plúmula $(\mathrm{cm})$ se midió desde la intersección raíz-hipocótilo hasta la base del cotiledón (longitud de plúmula). Mientras que la longitud de la radícula $(\mathrm{cm})$ se midió desde la base del hipocótilo hasta el ápice de la radícula.

\section{Peso seco (miligramos por plántula)}

Las plántulas normales de cada unidad experimental se colocaron en bolsas de papel estraza perforadas e identificadas, que después se colocaron en una estufa de secado (RIOSA modelo $\mathrm{H}-24^{\circledR}$ ), 
a temperatura de $72{ }^{\circ} \mathrm{C}$ durante $24 \mathrm{~h}$. Al finalizar el tiempo se registró el peso de las muestras en una balanza analítica (AND modelo HR-200 ${ }^{\circledR}$ ), para determinar el valor de la biomasa seca y se reportó en miligramos por plántula.

\section{Diseño experimental y análisis estadístico}

El experimento se realizó mediante un diseño completamente al azar con siete tratamientos y tres repeticiones. La unidad experimental consistió en un rollo de papel anchor con 25 semillas. Para cumplir con los supuestos de normalidad, los datos de las variables porcentaje de germinación y de vigor se transformaron mediante la función arcoseno (Steel y Torrie 1962). Los resultados se presentaron como media \pm desviación estándar. Los datos de las variables se analizaron con ANOVA y las medias de tratamientos se compararon con la prueba de Tukey, con un nivel de significación de $5 \%(\alpha=0.05)$, se utilizó el paquete estadístico SAS versión 9.4.

\section{RESULTADOS}

\section{Síntesis de nanopartículas de hidroxiapatita}

Las HAp NPs se obtuvieron por reacción de precipitación química húmeda mediante agitación mecánica a 1000 rpm, el tamaño de las partículas resultó entre 10 y $45 \mathrm{~nm}$, sin embargo, el $53.1 \%$ de las NPs se encuentran en el rango de 15 a $25 \mathrm{~nm}$, con morfología predominantemente en forma de varilla, estas condiciones son deseables para su estudio y aplicación en la agricultura. En la Tabla 1 se muestra la distribución de tamaños y la morfología de las HAp NPs.

La Figura 1a muestra los patrones de difracción de rayos $\mathrm{X}$ del precipitado obtenido por agitación mecánica. Los resultados concuerdan con las respuestas típicas con mayor intensidad en los pi$\cos (002,211,112,300$ y 202), por lo que el polvo se caracteriza como hidroxiapatita pura, de acuerdo con la base de datos internacional JCDPS 9-432 (Figura 1b), por lo tanto, se confirma la formación de nHAp. También se observa que la agitación mecánica produce tamaños de cristalita de $15.2 \pm 1.3 \mathrm{~nm}$.
El análisis de la muestra en el microscopio TEM (Figura 2a), presenta la imagen de un nHAp sintetizado con agitación mecánica, y se observa una forma de varilla alargada de NPs. En la Figura $2 b$ se observa la distribución de los tamaños de HAp NPs después de medir más de 300 nanopartículas. La mayoría $(\sim 65 \%)$ de las NPs mostraron diámetros de 10 a $25 \mathrm{~nm}$. Solo $\sim 4 \%$ exhibieron tamaños superiores a $35 \mathrm{~nm}$. En la Figura $2 \mathrm{c}$ representativa de HRTEM, se aprecia un diámetro de la partícula con un valor medio de $19.13 \mathrm{~nm}$. Lo anterior concuerda con la frecuencia de tamaño promedio observada en el esquema del panel 2b. El patrón de difracción SAED obtenido del área observada en el panel 2a, se muestra en el panel 2d. Este patrón está formado por anillos irregulares asociados con los planos (211, 102, 201 y 200) de la estructura de HAp NPs.

\section{Efecto de las nanopartículas de HAp NPs en la germinación y vigor de las semillas}

Los resultados muestran que en ambas variables los tratamientos fueron similares al control, excepto en dosis altas donde se observan diferencias significativas $(p \leq 0.05)$ de estas variables asociadas con la calidad fisiológica de las semillas de $R$. sativus (Tabla 2). El vigor de semilla tuvo un decremento de $11.8 \%(82.00 \pm 5.14)$ a $500 \mathrm{mg} \mathrm{L}^{-1}$, en comparación con el control $(93.00 \pm 3.95)$. Para las concentraciones de $0,25,50,100,200$ y $1000 \mathrm{mg} \mathrm{L}^{-1}$ no se obtuvieron diferencias estadísticas significativas. En la variable germinación, la máxima disminución se encontró con $1000 \mathrm{mg} \mathrm{L}^{-1}$ (Tabla 2) con una reducción del $14.4 \%$ en comparación con el testigo $(90.0 \pm 5.64 \%)$. Para las concentraciones de 0,25 , $50,100,200$ y $500 \mathrm{mg} \mathrm{L}^{-1}$ no se obtuvieron diferencias estadísticas significativas.

\section{Longitud de plúmula y radícula}

Las nHAp no afectaron de forma significativa ( $p$ $\leq 0.05$ ) la longitud de plúmula como se muestra en la Tabla 2. Con la concentración de $200 \mathrm{mg} \mathrm{L}^{-1}$ se tuvo una longitud de $7.71 \pm 0.36 \mathrm{~cm}$, estadísticamente similar al tratamiento control $(7.04 \pm 0.33 \mathrm{~cm})$ con diferencia numérica del $8.7 \%$. Para la concentración de $25 \mathrm{mg} \mathrm{L}^{-1}$, las longitudes de las plúmulas fueron 
Tabla 1. Distribución de tamaños y morfología de nano hidroxiapatita.

\begin{tabular}{cccc}
\hline Intervalo de Diámetros $(\mathrm{nm})$ & Frecuencia Absoluta & $\%$ & Morfología \\
\hline$[10-15]$ & 43 & 12.2 & \\
{$[15-20]$} & 95 & 27.0 & \\
{$[20-25]$} & 92 & 26.1 & \\
{$[25-30]$} & 71 & 20.2 & \\
{$[30-35]$} & 36 & 10.2 & \\
{$[35-40]$} & 13 & 3.7 & \\
{$[40-45]$} & 2 & 0.6 & \\
SUMA & 352 & 100.0 & \\
\hline
\end{tabular}

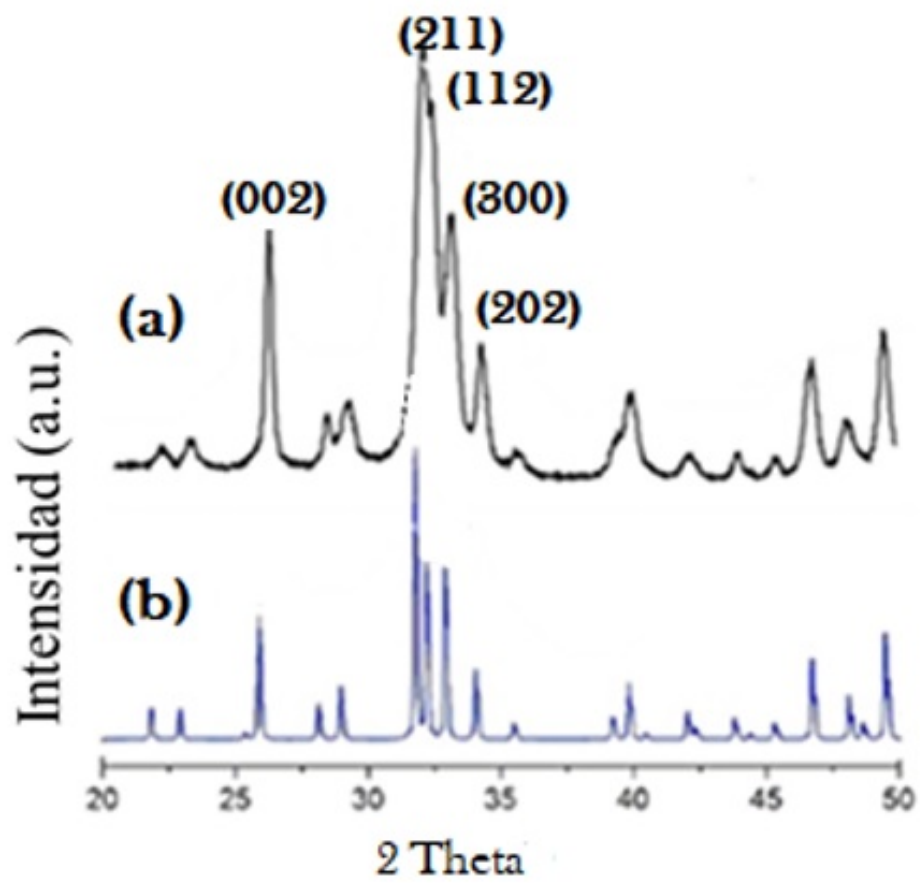

Figura 1. Patrones de difracción de DRX de la muestra de polvo de HAp NPs obtenido por dos métodos: (a) agitación mecánica y (b) el patrón de difracción estándar para HA según la carta JCPDS 9-432.

de $6.74 \pm 0.32 \mathrm{~cm}$ o $4.3 \%$ más cortas que el control $(7.04 \pm 0.33 \mathrm{~cm})$ sin ser estadísticamente diferentes. Los tratamientos de 0, 50, 100, 500 y $1000 \mathrm{mg} \mathrm{L}^{-1}$ fueron estadísticamente iguales. Con respecto a la variable crecimiento de la radícula, se observó un aumento con el tratamiento de $200 \mathrm{mg} \mathrm{L}^{-1}$ de $\mathrm{nHAp}$, alcanzó una longitud de $14.45 \pm 0.92 \mathrm{~cm}$, sin tener una diferencia estadística significativa en comparación con el control (13.43 $\pm 0.86 \mathrm{~cm})$; mientras que a $25 \mathrm{mg} \mathrm{L}^{-1}$ la longitud de radícula reportó $12.23 \pm$ $0.79 \mathrm{~cm}$, sin tener una diferencia estadística significativa contra el tratamiento control, pero apreciándose una disminución numérica del $8.9 \%$. Tanto para plú- mula como para radícula, se observaron diferencias estadísticas significativas entre los tratamientos de 25 y $200 \mathrm{mg} \mathrm{L}^{-1}$.

\section{Producción de biomasa seca}

En la Tabla 2, se presenta el efecto que tuvieron las HAp NPs en la variable producción de biomasa seca de plántulas. Las cantidades ahí mostradas revelan que la mayor acumulación de peso seco (8 $086 \pm 606.57 \mathrm{mg}$ plántula ${ }^{-1}$ ), se obtuvo cuando se aplicó la dosis de $1000 \mathrm{mg} \mathrm{L}^{-1}$, habiendo superado significativamente a los tratamientos que recibieron 0,50 y $200 \mathrm{mg} \mathrm{L}^{-1}$, ya que estos repor- 


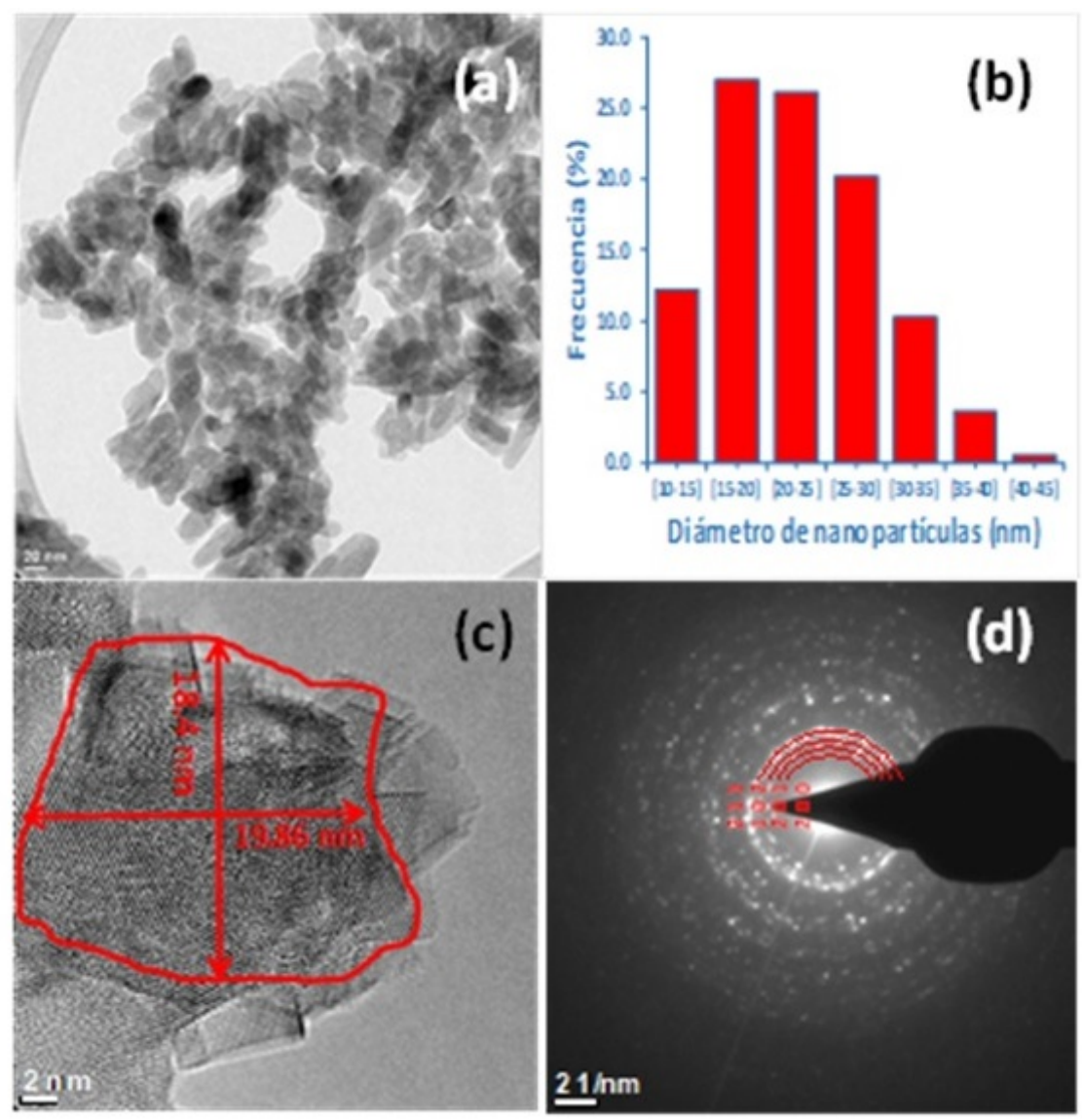

Figura 2. Micrografías correspondientes a la muestra de nanopartículas de hidroxiapatita sintetizadas: (a). Imagen TEM que muestra aglomerado de nanopartículas. (b). Distribución de tamaños de las NPs. (c). Imagen HRTEM de una nanopartícula. (d). Patrón de difracción SAED obtenido del área observada en la imagen (c).

Tabla 2. Comparación de medias para el porcentaje y vigor de germinación, así como para la longitud de vástago, de radícula y biomasa seca de plántulas de Rhapanus sativus tratadas con nanopartículas de hidroxiapatita.

\begin{tabular}{cccccc}
\hline Dosis aplicada & Germinación & Vigor de germinación & LP & LR & PS \\
\hline$\left(\mathrm{mg} \mathrm{L}^{-1}\right)$ & $(\%)$ & $(\%)$ & $(\mathrm{cm})$ & $(\mathrm{cm})$ & (mg plántula $\left.^{-1}\right)$ \\
\hline 0 & $90 \pm 5.64^{a}$ & $93 \pm 3.95^{a}$ & $7.0 \pm 0.33^{a b}$ & $13.4 \pm 0.86^{a b}$ & $6758 \pm 506.95^{b}$ \\
25 & $84 \pm 5.26^{a b}$ & $87 \pm 3.69^{a b}$ & $6.7 \pm 0.32^{b}$ & $12.2 \pm 0.79^{b}$ & $7102 \pm 532.75^{a b}$ \\
50 & $86 \pm 5.39^{a b}$ & $88 \pm 3.57^{a b}$ & $7.4 \pm 0.35^{a b}$ & $14.3 \pm 0.92^{a b}$ & $6813 \pm 511.07^{b}$ \\
100 & $87 \pm 5.45^{a b}$ & $87 \pm 3.69^{a b}$ & $7.5 \pm 0.35^{a b}$ & $14.0 \pm 0.92^{a b}$ & $7088 \pm 531.70^{a b}$ \\
200 & $87 \pm 5.45^{a b}$ & $88 \pm 3.74^{a b}$ & $7.7 \pm 0.36^{a}$ & $14.4 \pm 0.92^{a}$ & $6725 \pm 504.47^{b}$ \\
500 & $82 \pm 5.14^{a b}$ & $82 \pm 3.48^{b}$ & $7.4 \pm 0.35^{a b}$ & $14.3 \pm 0.92^{a b}$ & $7034 \pm 527.65^{a b}$ \\
1000 & $77 \pm 4.82^{b}$ & $87 \pm 3.69^{a b}$ & $7.0 \pm 0.33^{a b}$ & $14.2 \pm 0.92^{a b}$ & $8086 \pm 606.57^{a}$ \\
\hline
\end{tabular}

$\mathrm{LP}=$ Longitud de plúmula, $\mathrm{LR}=$ Longitud de radícula, $\mathrm{PS}=$ Peso seco. Los valores son el promedio de tres repeticiones. Medias $(n=3) \pm$ desviación estándar. Las diferentes letras son estadísticamente diferentes (Tukey, $p \leq$ $0.05)$ y representan las diferencias entre tratamientos.

taron $16.4,15.7$ y $16.8 \%$, respectivamente. Mientras que los tratamientos con las dosis de 25, 100, $500 \mathrm{y}$ $1000 \mathrm{mg} \mathrm{L}^{-1}$ no mostraron diferencias estadísticamente significativas.

\section{DISCUSIÓN}

\section{Síntesis de nanopartículas de hidroxiapatita}

Con respecto a la morfología, la agitación mecánica produjo nanopartículas en forma de varilla 
con tamaño entre 15 y $25 \mathrm{~nm}$. De acuerdo con Alvear et al. (2017), la velocidad de agitación influye en la obtención de partículas más pequeñas, debido a que el aumento de la velocidad de agitación incrementa el choque entre núcleos de reactivos de $\mathrm{NPs}$ de $\mathrm{Fe}_{3} \mathrm{O}_{4}$, por lo que se obtienen partículas pequeñas y uniformes. Por otro lado, Yusoff et al. (2018) reportaron que la agitación mecánica aumenta la energía del sistema, lo que aumenta los choques entre los núcleos de los reactivos debido a su óptima distribución en la solución, lo que mejora el rendimiento de la reacción y acelera la precipitación. Mientras que la estequiometria de la reacción indica que la totalidad de reactivos se convirtieron en nHAp, lo que concuerda con lo reportado por Yusoff et al. (2018) sobre la influencia de la agitación mecánica en el rendimiento de la reacción.

\section{Efecto de las nanopartículas de HAp en la germi- nación y vigor de las semillas}

Por otro lado, el efecto de distintos tipos de nanomateriales (metálicos o derivados del carbono) en el crecimiento de plantas, ha sido discutido por diversos autores como Liu y Lal (2015), quienes menciona que la germinación es una de las etapas más importantes para el establecimiento y rendimiento de los cultivos, por lo que es de gran interés conocer cómo actúan las NPs en estos procesos (GarcíaLópez et al. 2019). Pero es difícil establecer un modelo de respuesta de los efectos de las NPs en la fisiología de las semillas y plántulas, ya que el efecto depende de la especie, la concentración y el tipo de NPs (Lin y Xing 2007, Arruda et al. 2015). Mientras que otros estudios reportan que la aplicación de nHAp no influye en la germinación y crecimiento de plántulas.

Por ejemplo, Ortiz et al. (2017) indican que la aplicación de una concentración de $8000 \mathrm{mg} \mathrm{L}^{-1}$ de $\mathrm{nHAp}$ en $L$. sativa, equivalente a $247 \mathrm{mg} \mathrm{L}^{-1}$ de $P$, es fitotóxica; pero durante la etapa de imbibición de las semillas, las nHAp no causaron variación en el porcentaje de germinación entre el testigo y el tratamiento. Lo que sugiere que la concentración del $P$ disponible en las HAp NPs no fue suficiente para absorberse por las semillas de L. sativa. Por otra parte, Marchiol et al. (2019) y Ortiz et al. (2017) reportan que HAp NPs estabilizado con carboximetil celulosa, aplicada a plantas de tomate, no afecta el porcentaje de germinación bajo ninguna de las concentraciones de nHAp utilizadas. Por lo que los resultados del presente trabajo coinciden parcialmente con lo antes señalado, ya que no se observó un efecto significativo en los tratamientos de 25, 50 100, 200 y $500 \mathrm{mg} \mathrm{L}^{-1}$, con respecto al control, solo el tratamiento con $1000 \mathrm{mg} \mathrm{L}^{-1}$ presenta diferencia estadística significativa al reducirse en $14.4 \%$ la germinación con respecto al tratamiento control $(90.00 \pm$ 5.64). Por otra parte, la variable de vigor fue afectada con el tratamiento de $500 \mathrm{mg} \mathrm{L}^{-1}$ al reducirse $11.83 \%$ con respecto al tratamiento control. Aunque estas pequeñas reducciones no se pueden considerar como un efecto fitotóxico, sino como un efecto inhibidor del crecimiento relacionado con un elevado potencial osmótico por las dosis de HAp NPs (Hojjat et al. 2017). Pero este comportamiento pudiera ser atribuido a la diferencia en el tamaño y morfología de las nanopartículas utilizadas.

En relación con el tamaño y morfología de las NPs y su efecto biológico, Ortiz et al. (2017) utilizaron NPs con tamaños menores a $100 \mathrm{~nm}$ y morfología esférica, mientras que Marchiol et al. (2019) utilizaron NPs con tamaños entre 94 a 163.3 nm y morfología diversa, sin embargo, esos autores señalan que la forma de la nHAp es crítica para producir anomalías en pigmentos fotosintéticos de tomate. En el presente trabajo, las partículas sintetizadas presentaron un tamaño entre 10 y $45 \mathrm{~nm}$ con forma de varillas alargadas principalmente, estas características posiblemente facilitaron la penetración de las HAp NPs a través del tegumento de las semillas y provocar el efecto inhibidor en la germinación y el vigor. Churilov et al. (2019) reportan que al usar NPs de cobre con tamaños de partícula promedio de 45 y $200 \mathrm{~nm}$ encontraron que las NPs de $45 \mathrm{~nm}$, tuvieron mayor actividad biológica con respecto a la energía germinativa en semillas y plántulas de mostaza blanca.

La fitotoxicidad o inhibición de crecimiento y producción de biomasa relacionada con las HAp NPs no ha sido reportado en investigaciones previas, pero pudiera ser semejante al efecto biológico que cau- 
san las NPs metálicas en concentraciones elevadas. A este respecto García-López et al. (2018) señalan que la etapa de germinación de semillas es particularmente sensible a las condiciones de estrés inducidas por elevadas concentraciones de nanomateriales aplicados, debido a que pueden provocar fitotoxicidad e inhibición de crecimiento y producción de biomasa. Por su parte, Lee et al. (2010) y Manke et al. (2013), señalan que altas dosis de NPs metálicas inducen fitotoxicidad en la germinación y el vigor, principalmente por toxicidad, debido a la composición química de los nanomateriales, ya que liberan iones metálicos tóxicos, y por la inhibición fisiológica causada por la morfología, estructura, y/o tamaño de las NP.

Por lo tanto, el tamaño de las HAp NPs es un factor clave a considerar en futuras investigaciones que intenten describir su comportamiento fitotóxico en dosis elevadas. Por otro lado, la morfología de la semilla también tiene una función muy importante, ya que los pliegues en el tegumento de la semilla pueden ayudar a que las NPs se fijen y se mejore así la posible interacción con éstas, al aumentar la atracción electrostática y las interacciones hidrofóbicas en la superficie de las semillas, lo que puede influir en su fitotoxicidad (Wu et al. 2012). En relación con lo anterior, Jain et al. (2017) al evaluar la fitotoxicidad de NPs de ZnO en semillas de mijo perla (Pennisetum glaucum), tomate y trigo, reportan que una concentración de $1000 \mathrm{mg} \mathrm{L}^{-1}$ redujo su germinación en 18, 30 y $60 \%$, respectivamente. Estos resultados tan desiguales con respecto a la especie vegetal, pudieran atribuirse a que el menor efecto en las semillas del mijo perla se debió a que tienen una cutícula gruesa y testa lisa, lo que las hace menos sensible a las NPs ZnO en comparación con las semillas de trigo y tomate, las cuales tienen una capa delgada y testa rugosa. La reducción porcentual de germinación del mijo perla antes señalada, resulta ser similar a la obtenida con el rábano del presente trabajo. Estos efectos análogos se pueden explicar por una posible similitud entre los dos tipos de semilla, especialmente con respecto a su morfología, cutícula y testa.

\section{Longitud de plúmula y radícula}

Con respecto a los efectos en las plántulas, los resultados muestran que las bajas concentraciones de HAp NPs $\left(25 \mathrm{mg} \mathrm{L}^{-1}\right)$ redujeron la elongación de plúmula y radícula, mientras que la concentración de $200 \mathrm{mg} \mathrm{L}^{-1}$ promovió el crecimiento de esas variables; en cambio las dosis de 50, 100, 500 y $1000 \mathrm{mg} \mathrm{L}^{-1}$ no afectaron significativamente el crecimiento. Un posible efecto estimulante en el crecimiento se puede deber a la penetración de las HAp NPs en las semillas, lo que permite la imbibición de $\mathrm{P}$ y $\mathrm{Ca}$, así como de agua y micronutrientes, con una consecuente movilización de reservas que apoyan las primeras etapas del proceso de germinación (Lin y Xing 2007); además, por activación de enzimas como la amilasa $\alpha$ y $\beta$ que son las responsables de las reacciones metabólicas en la etapa de germinación (Krishna y Natarajan 2014).

Por otra parte, el nivel de ácido indolacético en las raíces y los brotes podría haberse incrementado, lo que a su vez aumenta la germinación, el vigor y el crecimiento de las plántulas (García-López et al. 2018). Mientras que Bala et al. (2014) al aplicar HAp NPs en Cicer arietinum, encontraron que la concentración que mostró un mayor crecimiento de brotes fue de $1000 \mathrm{mg} \mathrm{L}^{-1}$, mientras que para Marchiol et al. (2019) el mejor tratamiento fue de $500 \mathrm{mg} \mathrm{L}^{-1}$ : En ese contexto en el presente estudio no se detectaron diferencias significativas en el crecimiento de brotes de los tratamientos con respecto al control, por lo que, con los resultados de este bioensayo no es posible asegurar que las concentraciones evaluadas de las nHAp puedan ser usadas eficazmente como nanofertilizante.

\section{Producción de biomasa seca}

La dosis de $1000 \mathrm{mg} \mathrm{L}^{-1}$ de NPs HAp mejoró la producción de esta variable con respecto al tratamiento control, ya que se detectó una diferencia de $16.4 \%$, valor semejante al consignado por Liu y Lal. (2014), quienes aplicaron HAp NPs en soya (Glycine max) y encontraron que la biomasa seca aérea producida fue de $13 \mathrm{~g}$ por planta, en comparación con $11 \mathrm{~g}$ por planta lograda cuando se aplicó el fertilizante fosfatado. Estos resultados evidencian que las HAp NPs pueden ser utilizadas para mejorar algunas respuestas fisiológicas rela- 
cionadas con la producción de biomasa. Rafique et al. (2018) señalan que cada especie vegetal tiene un mecanismo fisiológico específico para la absorción de nutrientes como el fósforo, pero en este trabajo no se dilucidaron los mecanismos que utilizan las plantas de rábano para tomar HAp NPs como fuente de nutrientes, por lo que se requiere realizar investigación más sistemática en este sentido.

\section{CONCLUSIONES}

El uso de la agitación mecánica durante una reacción química, facilita los enlaces entre reactivos y mejora el rendimiento de la reacción para sintetizar HAp NPs, ya que este tratamiento físico permite romper agregados y generar NPs de hidroxiapatita de tamaños reducidos entre 10 y $45 \mathrm{~nm}$. La caracterización microscópica realizada demostró que la síntesis de HAp NPs, mediante un proceso químico sencillo, podría ser escalado al nivel industrial para producir a bajo costo nanomateriales con uso potencial en el sector agropecuario. Las HAp NPs sin- tetizadas y aplicadas en dosis bajas a las semillas de rábano no afectan el vigor y la germinación, pero dosis de 500 y $1000 \mathrm{mg} \mathrm{L}^{-1}$ generan un efecto inhibitorio de esta y otras variables. Para el tamaño de plúmula y radícula, no se tuvieron efectos estadísticos significativos, mientras que para la acumulación de biomasa seca se apreció un incremento con la dosis de $1000 \mathrm{mg} \mathrm{L}^{-1}$, lo que indica que el rango de las concentraciones utilizadas de HAp NPs no estimularon estadísticamente algunos variables en las plántulas de rábano.

\section{AGRADECIMIENTOS}

Al proyecto No. 268 de Fronteras de la CienciaCONACYT, así como al Centro de Investigación de Química Aplicada (CIQA) por el apoyo otorgado al permitir que en sus instalaciones se realice este trabajo. Al Dr. Joelis Rodríguez Hernández, MC. Bertha A. Puente Urbina, MC. Beatriz Reyes Vielma y MC. Gladys de los Santos Villarreal por su valiosa ayuda.

\section{LITERATURA CITADA}

Alvear D, Galeas S, Guerrero VH, Debut A (2017) Synthesis and characterization of magnetite nanoparticles. Revista Politécnica 39: 61-66.

Antisari LV, Carbone S, Bosi S, Gatti A, Dinelli G (2018) Engineered nanoparticles effects in soil-plant system: Basil (Ocimum basilicum L.) study case. Applied Soil Ecology 123: 551-560.

Arruda SCC, Silva ALD, Galazzi RM, Azevedo RA, Arruda MAZ (2015) Nanoparticles applied to plant science: a review. Talanta 131: 693-705.

Bala N, Dey A, Das S, Basu R, Nandy P (2014) Effect of hydroxyapatite nanorod on chickpea (Cicer arietinum) plant growth and its possible use as nano-fertilizer. Iranian Journal of Plant Physiology 4: 1061-1069.

Banotra M, Kumar A, Sharma BC, Nandan B, Verma A, Kumar R, Gupta V, Bhagat S (2017) Prospectus of use of nanotechnology in agriculture-a review. International Journal of Current Microbiology and Applied Sciences 6: 1541.

Cordell D, White S (2015) Tracking phosphorus security: indicators of phosphorus vulnerability in the global food system. Food Security 7: 337-350.

Cordell D, Drangert JO, White S (2009) The story of phosphorus: Global food security and food for thought. Global Environmental Change 19: 292-305.

Churilov D, Churilova V, Stepanova I, Polischuk S, Gusev A, Zakharova O, Churilov G (2019) Size-dependent biological effects of copper nanopowders on mustard seedlings. IOP Conference Series: Earth and Environmental Science 392: 012008. Doi: 10.1088/1755-1315/392/1/012008. 
García-López JI, Niño-Medina G, Olivares-Sáenz E, Lira-Saldívar RH, Barriga-Castro ED, Vázquez-Alvarado R, Rodríguez-Salinas PA, Zavala-Garcia F (2019) Foliar application of zinc oxide nanoparticles and zinc sulfate boosts the content of bioactive compounds in habanero peppers. Plants 8(8): 254. Doi: 10.3390/plants8080 254

García-López JI, Zavala-García F, Olivares-Sáenz E, Lira-Saldívar RH, Díaz Barriga-Castro E, Ruiz-Torres NA, Ramos-Cortez E, Vázquez-Alvarado R, Niño-Medina G (2018) Zinc oxide nanoparticles boosts phenolic compounds and antioxidant activity of Capsicum annuum L. during germination. Agronomy 8: 215. Doi: 10.3390/agronomy8100215.

Hojjat SS, Kamyab M (2017) The effect of silver nanoparticle on Fenugreek seed germination under salinity levels. Russian agricultural sciences 43: 61-65.

ISTA (2004) International rules for seed testing. International Seed Testing Association. Zurich, Switzerland. 243p.

Izumi F, Takuji I (2015) Implementation of the Williamson-Hall and Halder-Wagner methods into RIETAN-FP. annual report. Advanced Ceeramics Research Center Nagoya Institute of Technology 3: 33-38.

Jain N, Bhargava A, Pareek V, Akhtar MS, Panwar J (2017) Does seed size and surface anatomy play role in combating phytotoxicity of nanoparticles? Ecotoxicology 26: 238-249.

Kabiri S, Andelkovic IB, Da Silva RC, Degryse F, Baird R, Tavakkoli E, McLaughlin MJ (2020) Engineered phosphate fertilizers with dual-release properties. Industrial \& Engineering Chemistry Research 59: 5512-5524.

Kottegoda N, Munaweera I, Madusanka N, Karunaratne V (2011) A green slow-release fertilizer composition based on urea-modified hydroxyapatite nanoparticles encapsulated wood. Current Science 101: 73-78.

Krishna KK, Natarajan N (2014) Customizing zinc oxide, silver, and titanium dioxide nanoparticles for enhancing groundnut seed quality. Indian Journal of Science and Technology 7: 1376-1381.

Lee CW, Mahendra S, Zodrow K, Li D, Tsai YC, Braam J, Alvarez PJ (2010) Developmental phytotoxicity of metal oxide nanoparticles to Arabidopsis thaliana. Environmental Toxicology and Chemistry: An International Journal 29(3): 669-675.

Lin D, Xing B (2007) Phytotoxicity of nanoparticles: inhibition of seed germination and root growth. Environmental Pollution 150: 243-250.

Liu R, Lal R (2014) Synthetic apatite nanoparticles as a phosphorus fertilizer for soybean (Glycine max). Scientific reports 4(1): 1-6. Doi: 10.1038/srep05686

Liu R, Lal R (2015) Potentials of engineered nanoparticles as fertilizers for increasing agronomic productions. Science of the Total Environment 514: 131139. Doi: 10.1016/j.scitotenv.2015.01.104

Manke A, Wang L, Rojanasakul Y (2013) Mechanisms of nanoparticle-induced oxidative stress and toxicity. BioMed research international 942916. Doi: 10.1155/2013/942916.

Marchiol L, Filippi A, Adamiano A, Degli Esposti L, lafisco M, Mattiello A, Braidot E (2019) Influence of Hydroxyapatite Nanoparticles on Germination and Plant Metabolism of Tomato (Solanum lycopersicum L.): Preliminary Evidence. Agronomy 9(4): 161. Doi: 10.3390/agronomy9040161.

Méndez-Argüello B, Lira-Saldivar RH (2019) Uso potencial de la zeolita en la agricultura sustentable de la nueva revolución verde. Ecosistemas y Recursos Agropecuarios 6: 191-193.

Montalvo D, McLaughlin MJ, Degryse F (2015) Efficacy of hydroxyapatite nanoparticles as phosphorus fertilizer in andisols and oxisols. Soil Science Society of America Journal 79(2). Doi: 10.3390/agronomy9040161 
Ortiz CG, Carrillo-González R, González-Chávez MCA, López LJ (2017) Síntesis de nanopartículas de hidroxiapatita y su toxicidad en lechuga (Lactuca sativa L.). AGROProductividad 10: 98-106.

Pazos-Rojas, LA, Marín-Cevada V, Elizabeth Y, García M, Baez A (2016) Uso de microorganismos benéficos para reducir los daños causados por la revolución verde. Revista lberoamericana de Ciencias 3: 72-85.

Predoi D, Ghita RV, Iconaru SL, Cimpeanu CL, Raita SM (2020) Application of nanotechnology solutions in plants fertilization. In: Solankey SS, Akhtar S, Luna Maldonado AI, Rodriguez-Fuentes H, Vidales Contreras JA, Márquez Reyes JM (ed) Urban horticulture-necessity of the future. IntechOpen. Springer Nature Switzerland. pp: 51-75.

Rafique R, Zahra Z, Virk N, Shahid M, Pinelli E, Park TJ, Kallerhoff J, Arshad M (2018) Dose-dependent physiological responses of Triticum aestivum $\mathrm{L}$. to soil applied $\mathrm{TiO}_{2}$ nanoparticles: Alterations in chlorophyll content, $\mathrm{H}_{2} \mathrm{O}_{2}$ production, and genotoxicity. Agriculture, ecosystems \& environment 255: 95-101.

Ramesh N, Moratti SC, Dias GJ (2018) Hydroxyapatite-polymer biocomposites for bone regeneration: A review of current trends. Journal of Biomedical Materials Research Part B: Applied Biomaterials 106: 2046-2057.

Rane M, Bawskar M, Rathod D, Nagaonkar D, Rai M (2015) Influence of calcium phos-phate nanoparticles, Piriformospora indica and Glomus mosseae on growth of Zea mays. Advances in Natural Sciences: Nanoscience and Nanotechnology 6(4): 1- 8. Doi: 10.1088/2043-6262/6/4/045014/pdf

Rizwan M, Ali S, Qayyum MF, Ok YS, Adrees M, Ibrahim M, Zia-Ur-Rehman M, Farid M, Abbas F (2017) Effect of metal and metal oxide nanoparticles on growth and physiology of globally important food crops: A critical review. Journal Hazard Mater 322(Pt A): 2-16.

Rui M, Ma C, Tang X, Yang J, Jiang F, Pan Y, Xiang Z, Hao Y, Rui Y, Cao W, Xing B (2017) Phytotoxicity of silver nanoparticles to peanut (Arachis hypogaea L.): physiological responses and food safety. ACS Sustainable Chemistry \& Engineering 5: 6557-6567.

Saleh TA (2020) Nanomaterials: Classification, properties, and environmental toxicities. Environmental Technology \& Innovation 20. Doi: 10.1016/j.eti.2020.101067.

Steel RGD, Torrie JH (1962) Principles and procedures of statistics. With special Reference to the Biological Sciences. McGraw-Hill Book Company. New York, NY, USA. 481p.

Taşkın MB, Şahin Ö, Taskin H, Atakol O, Inal A, Gune A (2018) Effect of synthetic nano-hydroxyapatite as an alternative phosphorus source on growth and phosphorus nutrition of lettuce (Lactuca sativa L.) plants. Journal of Plant Nutrition 41: 1148-1154.

Wu SG, Huang L, Head J, Chen DR, Kong IC, Tang YJ (2012) Phytotoxicity of metal oxide nanoparticles is related to both dissolved metals ions and adsorption of particles on seed surfaces. Journal of Petroleum \& Environmental Biotechnology 3(4): 126. Doi: 10.4172/2157-7463.1000126.

Yusoff AHM, Salimi MN, Jamlos MF (2018) Critical parametric study on final size of magnetite nanoparticles. IOP Conference Series: Materials Science and Engineering 318: 2-20. Doi: 10.1088/1757-899X/318/1/012020. 